\title{
OS SISTEMAS TUTORIAIS INTELIGENTES NO AMBIENTE DE APRENDIZAGEM FOCANDO A MEDIAÇÃO E A TROCA DE EXPERIÊNCIAS
}

SMART TUTORIAL SYSTEMS IN THE LEARNING ENVIRONMENT FOCUSING ON MEDIATION AND EXCHANGE OF EXPERIENCES

\author{
Ademir Avila \\ Laura Marisa Carnielo Calejon \\ Marlene Alves Dias
}

\begin{abstract}
Resumo
Neste trabalho, tratamos de forma sucinta o referencial teórico da presente pesquisa sobre a mediação e a troca de experiências entre professores e estudantes e entre estudantes quando se considera o enfoque histórico social de Vygotsky. Em seguida, apresentamos a metodologia da pesquisa, assim como o Sistema Tutorial Inteligente construído para avaliar como são tratados os possíveis níveis de ajuda entre os diferentes atores, para o caso do estudo de lógica de programação nos cursos superiores em Tecnologia de Informação - TI. Posteriormente, colocamos esse sistema em funcionamento e, finalmente, consideramos as soluções adequadas propostas pelos estudantes aos algoritmos definidos como parâmetros no ensino de lógica de programação e alguns resultados e conclusões que se obtém por meio dessa análise.
\end{abstract}

Palavras-chave: Mediação; Níveis de Ajuda; Software Educativo; Sistema Tutorial Inteligente; Enfoque Histórico Cultural.

\begin{abstract}
In this paper we succinctly approach the theoretical basis of the presented research on mediation and exchange of experiences between professors and students and students among themselves considering Vygotsky's social historical view. Next we present the research methodology, as well as the Smart Tutorial System built to evaluate the way possible levels of assistance between the different actors are dealt with, in the case of study of programming logics in higher courses in Information Technology - IT. Subsequently, we have put this system in operation and, finally, we have considered the appropriate solutions proposed by students to the algorithms defined as parameters in the teaching of programming logics, and some results and conclusions obtainable by means of such analysis.
\end{abstract}

Keywords: Mediation; Levels of Assistance; Educational Software; Smart Tutorial System; SocioHistorical Focus. 


\section{Introdução}

Esse trabalho é uma parte da pesquisa desenvolvida por um grupo multidisciplinar (Matemática, Psicologia e Informática) preocupado com as dificuldades encontradas pelos educadores no processo de ensino e aprendizagem das diferentes disciplinas. Nessa pesquisa estudamos mais precisamente as possibilidades de melhorar esse processo quando se consideram as trocas de experiências entre os estudantes. Para isso, escolhemos trabalhar com as noções de lógica de programação para os cursos de Tecnologia, por se tratar de um grupo de estudantes que estamos habituados a seguir.

Escolhida a disciplina de lógica de programação e o curso em que propusemos a experiência, enfatizamos nossas análises sobre a construção de algoritmos, que são importantes para a formação em informática, necessitam de conhecimentos matemáticos para que possam ser desenvolvidos e permitem a mediação do professor, do computador e a troca de experiências entre os estudantes do grupo pesquisado.

Como nossa escolha é possibilitar ao estudante do curso de tecnologia uma nova forma de trabalho que oportunize a troca de experiência, pareceu-nos mais conveniente trabalhar com lógica de programação. Consideramos ainda que o trabalho autônomo só seja possível quando os estudantes são capazes de aplicar seus conhecimentos quando são solicitados explicitamente ou não. Para isso, quando se consideram as competências específicas dos estudantes em relação à disciplina de lógica de programação, podemos supor que aquelas que permitem avançar em termos de conhecimentos mobilizáveis e disponíveis são as seguintes:

- Conhecer os diferentes conectores.

- Interpretar conceitos sobre algoritmos.

- Compreender e aplicar a abstração para elaborar algoritmos.

- Desenvolver a aplicação dos conceitos de Álgebra Booleana na construção de algoritmos.

Essas habilidades proporcionarão aos estudantes um trabalho autônomo, possibilitando trocas de experiências entre os diferentes pares que encontrarão em sua profissão e a aplicação de seus conhecimentos de maneira disponível quando for necessário.

Essas atitudes ultrapassam o aspecto da simples aplicação dos conhecimentos, exigindo, por parte do estudante - futuro profissional, uma responsabilidade de organização do trabalho pessoal considerando seu próprio desenvolvimento e de seus pares.

Verificamos que existem poucos trabalhos nesse sentido e que, tanto no ensino médio quanto no ensino superior, os estudantes têm grandes dificuldades na recepção/assimilação dos conhecimentos, pois muitas vezes não dispõem das competências e habilidades necessárias para o seu desenvolvimento escolar e 
profissional. Muitas dessas dificuldades estão associadas a não mobilização dos conhecimentos de Matemática e Tecnologia adquiridos no ensino fundamental. Isso os conduz a uma situação de desinteresse difícil de ser ultrapassada, uma vez que a falta de conhecimentos mobilizáveis tende a aumentar no decorrer das diferentes etapas da escolaridade.

A escolha referida nos induz a considerar um dos princípios básicos da teoria de Vygotsky (1988) que é o conceito de Zona de Desenvolvimento Proximal - ZDP, que representa a diferença entre a capacidade do estudante de resolver problemas por si mesmo e a capacidade de resolvê-los com ajuda de alguém. A Zona de Desenvolvimento Proximal - ZDP abrange assim todas as funções e atividades que o estudante consegue desempenhar apenas se houver ajuda de alguém, sendo que a pessoa que intervém para orientar o estudante pode ser o professor, os pais e até mesmo um amigo que já tenha desenvolvido a habilidade requerida.

Além disso, de acordo com Vygotsky (1988), uma característica essencial do aprendizado é que ele desperta vários processos de desenvolvimento internamente, os quais funcionam apenas quando o estudante interage em seu ambiente de convívio.

Considerando as abordagens mencionadas e a importância da troca de conhecimento para o desenvolvimento dos estudantes, inicia-se este trabalho com os seguintes questionamentos:

1) Quais conhecimentos de lógica de programação são necessários para compreender e desenvolver algoritmos adequados e eficientes?

2) A proposta de Vygotsky (1988) aliada a um sistema tutorial inteligente pode ser um recurso tecnológico adequado para o ensino de lógica de programação?

3) A mediação e a troca de experiências entre o professor e os estudantes pode ser um fator de desenvolvimento do conhecimento do grupo?

4) Todos esses recursos utilizados de maneira adequada podem auxiliar no nivelamento do conhecimento do grupo?

$\mathrm{Na}$ tentativa de responder a tais questionamentos, foi construído e implementado um Sistema Tutorial Inteligente - STI, denominado Portal Lógica de Programação, atrelado à proposta de Vygotsky. Desta forma, verificamos por meio desse recurso tecnológico os diferentes níveis de conhecimento esperados dos estudantes ao desenvolver atividades associadas às noções a serem desenvolvidas na disciplina de lógica de programação. Essa análise foi feita por meio de uma pesquisa com grupos de estudantes dos cursos de Ciência da Computação e Tecnologia de Informação, usando o recurso tecnológico citado anteriormente. Na sequência apresentamos uma breve descrição do referencial teórico utilizado na pesquisa.

\section{Referencial Teórico da Pesquisa}


Para viabilizar a proposta já referida, propusemo-nos a trabalhar a questão da aprendizagem segundo a proposta de Vygotsky (1988) e abordar mais especificamente as questões associadas aos conteúdos de lógica de programação.

Sendo assim, a presente pesquisa visou disponibilizar um recurso tecnológico num ambiente de aprendizagem com base na proposta de Vygotsky (1988), isto é, num ambiente que permitisse que o próprio estudante desenvolvesse seu conhecimento uma vez que o ambiente proporciona tanto acesso ao seu conhecimento individual, como também o conhecimento do grupo, em um contexto de mediação que envolve a interação com o professor, com o Sistema Tutorial Inteligente - STI, com as respostas do próprio estudante e as respostas dadas pelos colegas.

A proposta de um ambiente de aprendizagem (interativo) utilizando recursos tecnológicos pode acarretar a melhor adaptação do nível de conhecimento inicial dos estudantes, isto é, eles podem utilizar conhecimentos mais próximos de suas Zonas de Desenvolvimento Proximal - ZDP e as interações entre os estudantes com o sistema, que é constituído de atividades pré-definidas com objetivos bem determinados para que o saber em jogo na atividade possa tornar-se um novo objeto do conhecimento dos estudantes e, consequentemente, um novo conceito agregado que favorece um avanço.

É por meio dessa leitura do desenvolvimento dos estudantes dada por Vygotsky que se verificou até onde os estudantes são capazes de realizar um trabalho autônomo, quais os estímulos e mediações necessários para ajudá-los a avançar e se 0 ambiente como foi idealizado possibilitou realmente 0 desenvolvimento esperado dos estudantes.

Com o objetivo de abordar o pensamento de Vygotsky (1988) aliado aos recursos de tecnologia, propusemos verificar se eles podem auxiliar o professor em suas atividades didáticas. Assim, analisamos a importância do trabalho cooperativo no processo de ensino e aprendizagem, utilizando ambientes com Sistemas Tutoriais Inteligentes - STI, onde a interação, a mediação e a ação são fundamentais para que a aprendizagem do estudante se concretize. (CURILEM e AZEVEDO, 2003). Foi escolhida a teoria de Vygostsky (1988), pois entre outros conceitos, ela aborda a mediação e a troca de experiências para promover a Nova Zona de Desenvolvimento Real - ZDR.

Como o principal objetivo de um professor é o desenvolvimento da aprendizagem do estudante, e para atingir esse objetivo não basta somente dar uma boa aula, trabalhar bem os conteúdos, mas deve-se ter bem claras as concepções teóricas que fundamentam a sua prática, entende-se que a participação do professor, enquanto mediador de conhecimento, sendo presencial e/ou virtual, é de muita importância para o cumprimento de objetivo de ensinar, e se o professor tiver recursos que o auxiliem, mais proveitoso e eficiente será esse desenvolvimento de aprendizagem. 
Observamos ainda que a aprendizagem é um processo pelo qual o ser humano apropria-se do saber produzido pela sociedade. Em qualquer ambiente, a aprendizagem é um processo ativo que conduz a transformações no homem. (MOURA, AZEVEDO, MEHLECKE, 2005)

Além disso, são as transformações sociais, econômicas e tecnológicas que estabelecem novas formas de convívio e, evidentemente, de ensinar e aprender. Desse modo, as tecnologias da informação estão se consolidando e se ampliando no processo de ensino e aprendizagem como recurso de mediação entre o indivíduo e o conhecimento.

Vale lembrar ainda que a história e a evolução corrente do uso de computadores no ambiente educacional são discutidas em termos do desenvolvimento e teorias psicológicas. Essa história e evolução de ensino "artificial" podem ser vistas como uma integração entre a tecnologia disponível e a teoria de aprendizagem, interligadas pela aplicação didática dos programas computadorizados (LIMA, 2004).

Dos mais clássicos softwares educacionais, tutoriais, prática, simulação e jogos, aos tipos mais sofisticados, como realidade virtual, os projetos contêm, consciente ou inconscientemente, uma opção teórica de ensino e aprendizagem que privilegia o produto (SILVA, 2006).

As considerações acima nos levaram a escolher para a pesquisa o desenvolvimento de um ambiente para o ensino e aprendizagem de lógica de programação, com base na teoria de Vygotsky (1988) aliada aos recursos tecnológicos atualmente disponíveis no mercado, construindo assim um STI, o Sistema Tutorial Inteligente Portal Lógica de Programação - STIPLP.

Com isso, esperávamos que o estudante e o professor pudessem perceber mais rapidamente as dificuldades que aparecem durante o desenvolvimento das tarefas propostas e que a mediação do professor ou de outros estudantes servisse para o desenvolvimento individual e, assim, fizesse cada estudante progredir de forma autônoma encontrando respostas adequadas às diferenças individuais, pois os alunos estavam em diferentes Zonas de Desenvolvimento Proximal para os novos conhecimentos que se desejava introduzir, e os alunos mais adiantados poderiam também servir de mediador.

\section{Metodologia da Pesquisa}

Neste item foram descritos os procedimentos utilizados para o teste do ambiente de aprendizagem utilizando o STIPLP, aplicado aos estudantes dos cursos de Tecnologia e Computação de um Centro Universitário de São Paulo, visando verificar se o protótipo desenvolvido teria eficiência na comunicação e interface com os estudantes e, em um segundo momento, se o ambiente de aprendizado proposto melhorou o desempenho dos estudantes no que se refere à mediação $\mathrm{e}$ à troca de informações entre o grupo. 


\section{Aplicação do Sistema Tutorial Inteligente - STI}

Além das questões elaboradas no questionário para a verificação da utilização e ambiente de aprendizagem proposto pelo STIPLP, foi realizado outro tipo de coleta de dados, na mesma base de conhecimento, e com foco nas soluções propostas pelos estudantes. Esse tipo de coleta permite acompanhar se a troca de conhecimento de cada estudante pode ser realizada utilizando a ferramenta proposta, o STIPLP, considerando a ênfase dada por Vygotsky (1988) e as interpretações de Beatón (2005). Para isso, selecionamos o tópico Laço de Repetição, que faz parte dos tópicos abordados na construção de algoritmos, e o conhecimento que os estudantes têm de Comandos de Decisão e Definição de Variáveis de Memória.

Essa coleta foi dividida em quatro etapas, e as suas atividades estão descritas a seguir:

- Etapa 1: elaborar um algoritmo para realizar uma tarefa de contagem, em que os alunos só tiveram acesso à sua base de conhecimento inicial, seu conhecimento prévio;

- Etapa 2: elaborar um algoritmo que conte as horas de um dia completo, já nesta etapa os alunos tanto utilizaram seu conhecimento prévio como também acessaram o STIPLP como material de apoio para nos novos conceitos apresentados;

- Etapa 3: construir um algoritmo para identificar os números pares num intervalo previamente informado, e a cada etapa os alunos tiveram acesso a novos recursos; nesta etapa eles tiveram a mediação do professor;

- Etapa 4: elaborar um algoritmo para identificar números primos num intervalo previamente informado. Nessas etapas, utilizavam-se os conhecimentos desenvolvidos nas etapas anteriores; nesta etapa os alunos tiveram acesso à sua base de conhecimento inicial, o STIPLP, à mediação do professor e agora acesso à base de conhecimento do grupo, conforme figura 1:

\begin{tabular}{|l|c:c|c|c|}
\hline & Etapa 1 & Etapa 2 & Etapa 3 & Etapa 4 \\
\hline Acesso à Base de Conhecimento Individual & Sim & Sim & Sim & Sim \\
\hline Acesso ao Tutorial do STIPLP & Não & Sim & Sim & Sim \\
\hline Mediacáo do Professor & Náo & Náo & Sim & Sim \\
\hline Acesso à Base de Conhecimento do Grupo & Náo & Não & Não & Sim \\
\hline
\end{tabular}

Figura 1 - Tipo de Acesso de cada Etapa.

\section{Resultados e Conclusão}

Com os dados coletados, foi possível verificar que a cada uma dessas etapas a Zona de Desenvolvimento Proximal - ZDP do grupo foi ampliada, conforme o gráfico 1. Se considerarmos apenas os estudantes que apresentaram apenas uma solução adequada, temos os seguintes dados: Etapa 1: 3 estudantes (13\%); Etapa 2: 10 estudantes (43\%); encontramos um aumento de $30 \%$ nas soluções adequadas armazenadas na base de conhecimento do grupo. As mesmas análises para as Etapas 2 e 3 permitem considerar um aumento de $31 \%$ e para as 
Etapas 3 e 4, o aumento atingiu 26\%. O gráfico enseja avaliar as outras condições:

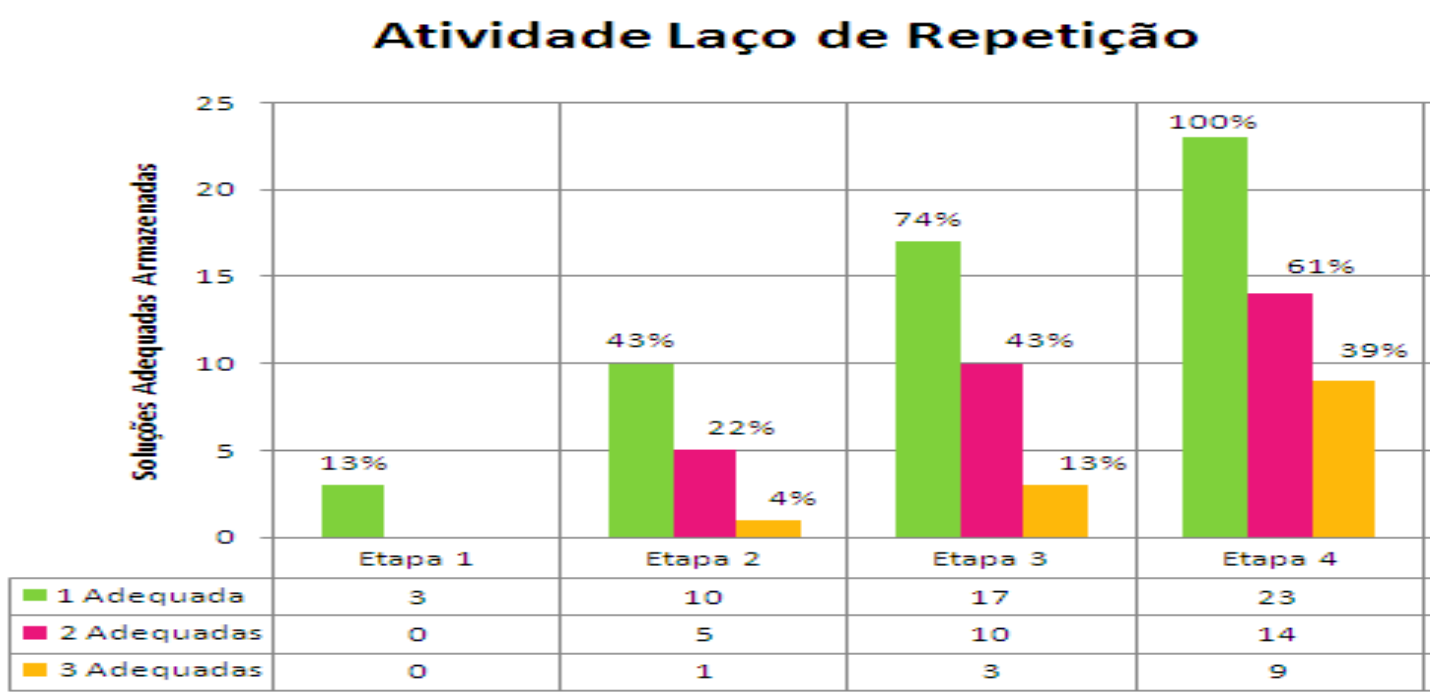

Gráfico 1 - Pesquisa Sobre a Troca de Informações na Atividade de Laço de Repetição.

Após esta análise, percebemos que os STls são recursos tecnológicos bastante úteis no ambiente de aprendizagem, e, neste caso, em especial no ensino de lógica de programação, e ainda podendo ser utilizados para outras áreas afins, possibilitando uma maior gama de opções, tanto para os estudantes, como também para os professores no que se refere aos ambientes de aprendizado.

\section{Referências}

BEATÓN, G. A. La Persona en lo Histórico Cultural. São Paulo: Linear B, 2005.

CURILEM, G M. S.; AZEVEDO, F. Didactic Ergonomy for the Interface of Intelligent Tutoring Systems. In: Computers and Education: Towards a Lifelong Learning Society. Netherlands: Dordrecht, Kluwer Academics Publishers, The Netherlands, 2003.

NUNES, I. D.; GATO, C. Modelagem e implementação do SEL - Software de Ensino de Lógica Proposicional. In: Anais do Il Simpósio de Informática da Região Centro / RS - Santa Maria, 2003.

LIMA, D. R. Uso da FAQ como Base de Casos em um Sistema Tutor Inteligente. 2004. Dissertação (Mestrado em Ciência da Computação) Universidade de Santa Catarina, Florianópolis, 2004.

MORAES, C. R. Estrutura de Dados e Algoritmos. São Paulo: Editora Berkely, 2001. 
MOURA, A. M. M.; AZEVEDO, A. M. P.; MEHLECKE, Q. As Teorias de Aprendizagem e os Recursos da Internet Auxiliando o Professor na Construção do Conhecimento. 2005. Disponível em:

<http://www.uel.br/seed/nte/as_teorias_de_aprendizagem_e_a_internet.htm> Acesso em: 05 set. 2007.

SILVA, A. P. C. Aplicações de Sistemas Tutores Inteligentes na Educação a Distância: Possibilidades e Limites. In: Anais do Seminário Nacional ABED de Educação a Distância. Brasília: ABED, 2006.

SUMMERVILLE, I. Engenharia de Software. Roger S. Pressman: Makron, 2003 VYGOTSKY, L. Pensamento e Linguagem. São Paulo: Martins Fontes, 1988. 1988. . Linguagem, Desenvolvimento e Aprendizagem. São Paulo, Icone, A Formação Social da Mente. 6. ed. São Paulo: Martins Fontes, 1998. 